\section{Tratamiento con estatinas en pacientes pediátricos con síndrome nefrótico resistente a esteroides. Reporte de dos casos}

\author{
Sánchez-García $C^{1}$, Bailón-Ortega $A A^{2}$, Zaltzman-Girshevich $S^{3}$
}

\begin{abstract}
Resumen
El síndrome nefrótico se define por la asociación de proteinuria, hipoalbuminemia, hiperlipidemia y edema. El $80 \%$ de los pacientes pediátricos con síndrome nefrótico primario responde a la terapia con esteroides; el 20\% restante requiere asociar otros medicamentos para alcanzar la remisión (ciclofosfamida, ciclosporina). La hiperlipidemia en el síndrome nefrótico es debida tanto a un incremento en la síntesis acompañada de disminución en la eliminación de los lípidos de la sangre; siendo la causa directa de esto la proteinuria. La hiperlipidemia incrementa el riesgo cardiovascular, así como el daño glomerular. Con base en esto, la hiperlipidemia persistente en el síndrome nefrótico resistente a esteroides debe ser tratada. Los inhibidores de la 3-hidroxi-3-metilglutaril-coenzima A (HMG-CoA) reductasa han demostrado efecto antiinflamatorio, inmunomodulador y antiproliferativo. Por ello el papel de las estatinas en el síndrome nefrótico va más allá de su efecto hipolipemiante.

Presentamos dos casos de pacientes pediátricos con diagnóstico de síndrome nefrótico resistente a esteroides y su evolución durante el tratamiento con ciclosporina y estatinas.
\end{abstract}

PALABRAS CLAVE: proteinuria, estatinas, síndrome nefrótico, hiperlipidemia.

Acta Pediatr Mex. 2017 Jan;38(1):26-32.

\section{Statins treatment in pediatric patients with steroid resistant nephrotic syndrome. A two case reports.}

Sánchez-García $C^{1}$, Bailón-Ortega $A A^{2}$, Zaltzman-Girshevich $S^{3}$
${ }^{1}$ Residente de Nefrología Pediátrica, Departamento de Nefrología Pediátrica, Instituto Nacional de Pediatría.

2Jefe de Nefrología Pediátrica, Hospital de la Niñez Oaxaqueña.

${ }^{3}$ Profesor Titular de Nefrología Pediátrica, Instituto Nacional de Pediatría.

Recibido: 15 de marzo del 2016

Aceptado: 28 de septiembre del 2016

Correspondencia

Carlos Sánchez García

sanchez.carlos8516@gmail.com

Este artículo debe citarse como

Sánchez-García C, Bailón-Ortega AA, ZaltzmanGirshevich S. Tratamiento con estatinas en pacientes pediátricos con síndrome nefrótico resistente a esteroides. Reporte de dos casos. Acta Pediatr Mex. 2017;38(1):26-32. 
remission (cyclophosphamide, cyclosporine). The hyperlipidemia in the nephrotic syndrome is due both to an increased synthesis and decreased elimination of blood lipids; being the direct cause of this the proteinuria. The hyperlipidemia increases cardiovascular risk and glomerular damage. Based on this, the persistent hyperlipidemia in the steroid-resistant nephrotic syndrome should be treated. The HMG-CoA reductase inhibitors have shown immunomodulatory, antiinflammatory and anti-proliferative effect. Therefore, the role of statins in the nephrotic syndrome goes beyond their lipid-lowering effect.

We present two cases of pediatric patients diagnosed with steroidresistant nephrotic syndrome and its evolution during treatment with cyclosporine and statins.

KEYWORDS: proteinuria; statins; nephrotic syndrome; hyperlipidemia

\author{
${ }^{1}$ Residente de Nefrología Pediátrica, Depar- \\ tamento de Nefrología Pediátrica, Instituto \\ Nacional de Pediatría. \\ 2Jefe de Nefrología Pediátrica, Hospital de \\ la Niñez Oaxaqueña. \\ ${ }^{3}$ Profesor Titular de Nefrología Pediátrica, \\ Instituto Nacional de Pediatría.
}

\section{Correspondence}

Carlos Sánchez García

sanchez.carlos8516@gmail.com

\section{INTRODUCCIÓN}

El síndrome nefrótico se define por la concurrencia de proteinuria, hipoalbuminemia, hiperlipidemia y edema. ${ }^{1}$ Más de $80 \%$ de los pacientes pediátricos con síndrome nefrótico primario remiten durante las primeras 4 a 8 semanas de tratamiento con esteroides con buena evolución a largo plazo; sin embargo, el resto de los pacientes presenta riesgo alto de desarrollar enfermedad renal crónica. ${ }^{2}$

Existen discrepancias en cuanto al tratamiento del síndrome nefrótico corticorresistente; se han utilizado diferentes opciones terapéuticas: pulsos de metilprednisolona, ciclofosfamida, levamisol y clorambucilo con una respuesta entre 50 y $70 \%{ }^{2,3}$ La terapia combinada de ciclosporina y prednisona induce remisión completa en algunos pacientes con síndrome nefrótico resistente a esteroides..$^{4,5}$

La hiperlipidemia en el síndrome nefrótico es debida tanto a un incremento en la síntesis acompañada de disminución en la eliminación de los lípidos de la sangre; siendo la causa directa de esto la proteinuria y no el aumento en la síntesis de albúmina..$^{6,7}$ El riesgo cardiovascular y la progresión del daño glomerular se perpetua ante la persistencia de hiperlipidemia. ${ }^{8}$ La persistencia prolongada de estos factores en pacientes con síndrome nefrótico corticorresistente es preocupante; motivo por el que, en el caso de síndrome nefrótico, debe de ser tratada. ${ }^{9}$

Los inhibidores de la 3-hidroxi-3-metilglutarilcoenzima A (HMG-CoA) reductasa (estatinas) han demostrado su efectividad y seguridad en el tratamiento de la hiperlipidemia en niños y adolescentes. ${ }^{10}$ Incluso se ha descrito su uso en pacientes con hiperlipidemia secundaria a patología renal, con disminución significativa de los niveles séricos de lípidos. ${ }^{11}$ Estos medicamentos han demostrado efecto antiinflamatorio, inmunomodulador y antiproliferativo; ${ }^{12}$ por ello el papel de las estatinas en el síndrome nefrótico va más allá de su efecto hipolipemiante.

Presentamos dos casos de pacientes pediátricos con diagnóstico de síndrome nefrótico resistente a esteroides y su evolución durante el tratamiento con ciclosporina y estatinas. 


\section{CASO CLÍNICO 1}

Niño de 9 años de edad con historia previa de 5 meses con edema facial intermitente. Ingresó con anasarca, síndrome nefrótico activo; se le dio tratamiento sintomático con furosemida y espironolactona. Permaneció 10 días hospitalizado, sin datos clínicos o de laboratorio que indicaran causas secundarias del síndrome nefrótico. Se inició tratamiento con prednisona $40 \mathrm{mg}$ diarios (60 mg/m²SC/día) y egresó. Recibió tratamiento únicamente por 7 días debido a celulitis de pared abdominal, acompañada de anasarca, derrame pleural bilateral y líquido de ascitis. Ingresó nuevamente y se le dio tratamiento con restricción hidrosalina, furosemida y espironolactona, así como clindamicina y ceftriaxona teniendo una evolución favorable. Egresó a los 23 días de hospitalización con: creatinina sérica $0.5 \mathrm{mg} / \mathrm{dL}$, albúmina $1 \mathrm{~g} / \mathrm{dL}$, colesterol $726 \mathrm{mg} / \mathrm{dL}$, triglicéridos $628 \mathrm{mg} / \mathrm{dL}$; excreción urinaria de proteínas de $418 \mathrm{mg} / \mathrm{kg} /$ día, relación proteínas-creatinina urinaria (PrU/CrU) $28.3 \mathrm{mg} / \mathrm{mg}$.

Se inició prednisona a dosis de $60 \mathrm{mg} / \mathrm{m}^{2} \mathrm{SC}$ /día. A las ocho semanas de tratamiento se encontró incremento de la albúmina sérica a $3.3 \mathrm{~g} / \mathrm{dL}$ pero los niveles de triglicéridos y colesterol, aunque disminuyeron, se encontraron aún por arriba del valor normal para la edad en 140 y 263 mg/dL, respectivamente; así mismo, una excreción de proteínas en orina de $88.4 \mathrm{mg} / \mathrm{kg} / \mathrm{d}$ ía, PrU/CrU $4.7 \mathrm{mg} / \mathrm{mg}$, aún en rango nefrótico, propiciaron que se clasificara como corticorresistente.

Se agregó ciclosporina 50 mg dos veces al día ( $5 \mathrm{mg} / \mathrm{kg} /$ día) con lo que la proteinuria disminuyó a 44 mg/kg/día a las 4 semanas (PrU/CrU 1.8 mg/mg) debajo del rango nefrótico; sin embargo, los valores de triglicéridos y colesterol permanecían aumentados en 96 mg/dL y 209 mg/dL. Previa firma de consentimiento informado se inició atorvastatina $10 \mathrm{mg}$ diarios, se mantuvo la dosis de ciclosporina y se inició reducción de esteroi- de, alcanzando al primer mes niveles normales de colesterol y, al segundo, niveles normales de triglicéridos (Cuadro 1).

A seis meses de seguimiento con: prednisona $5 \mathrm{mg}$ diarios, ciclosporina $50 \mathrm{mg}$ dos veces al día y atorvastatina $10 \mathrm{mg}$ al día, se encontró excreción urinaria de proteínas de $2.1 \mathrm{mg} / \mathrm{kg} /$ día; PrU/CrU $0.11 \mathrm{mg} / \mathrm{mg}$, albúmina 3.9 g/dL, colesterol $104 \mathrm{mg} / \mathrm{dL}$ y triglicéridos $52 \mathrm{mg} / \mathrm{dL}$.

El uso de estatinas tiene el riesgo de producir rabdomiolisis y elevación de los niveles de creatina fosfoquinasa (CPK); durante el tratamiento se supervisaron las concentraciones y no hubo elevación significativa respecto del valor basal. No se presentó evidencia de efectos adversos por el tratamiento ni recaídas en el síndrome nefrótico (función renal conservada) durante el seguimiento de 6 meses (Figura 1).

\section{CASO CLÍNICO 2}

Niño de 5 años de edad con diagnóstico de síndrome nefrótico corticorresistente y glomeruloesclerosis focal y segmentaria por biopsia renal desde los 2 años de edad, sin evidencia de etiología secundaria. Desde su diagnóstico presentó mala evolución con persistencia de la hiperlipidemia, hipoalbuminemia y proteinuria a pesar de tratamiento con esteroide y ciclosporina; tuvo múltiples internamientos por edema e infecciones frecuentes.

A tres meses del diagnóstico se le admitió en el servicio de nefrología pediátrica por un cuadro infeccioso de vías respiratorias superiores y anasarca, se restringió el aporte hídrico y se le dio tratamiento con furosemida y espironolactona; se disminuyó la inmunosupresión hasta remitir el proceso infeccioso. Evolucionó con disminución del edema y resolución del cuadro respiratorio, encontrándose con albúmina sérica $0.8 \mathrm{~g} / \mathrm{dL}$, colesterol 2,566 mg/dL, triglicéridos 2,834 mg/dL 
Sánchez-García C et al. Tratamiento con estatinas en síndrome nefrótico

Cuadro 1. Valores de laboratorio del caso clínico 1

\begin{tabular}{|c|c|c|c|c|c|}
\hline & $\begin{array}{c}\text { Relación PrU/CrU } \\
\text { (mg/mg) }\end{array}$ & $\begin{array}{l}\text { Proteinuria } \\
\text { (mg/kg/día) }\end{array}$ & $\begin{array}{l}\text { Albúmina sérica } \\
(\mathrm{g} / \mathrm{dL})\end{array}$ & $\begin{array}{l}\text { Triglicéridos } \\
\text { (mg/dL) }\end{array}$ & $\begin{array}{c}\text { Colesterol } \\
(\mathrm{mg} / \mathrm{dL})\end{array}$ \\
\hline Previo a tratamiento & 28.30 & 418.3 & 1.0 & 628 & 726 \\
\hline Esteroide (8 semanas) & 4.70 & 88.4 & 3.3 & 140 & 263 \\
\hline Esteroide + ciclosporina & 1.80 & 44.36 & 3.6 & 96 & 209 \\
\hline Estatina mes 1 & 1.21 & 22.49 & 3.7 & 141 & 140 \\
\hline Estatina mes 2 & 0.64 & 11.2 & 3.8 & 78 & 166 \\
\hline Estatina mes 3 & 0.31 & 5.98 & 4.0 & 85 & 139 \\
\hline Estatina mes 4 & 0.22 & 3.68 & 4.5 & 87 & 131 \\
\hline Estatina mes 5 & 0.17 & 2.7 & 4.1 & 267 & 179 \\
\hline Estatina mes 6 & 0.11 & 2.1 & 3.9 & 52 & 104 \\
\hline
\end{tabular}

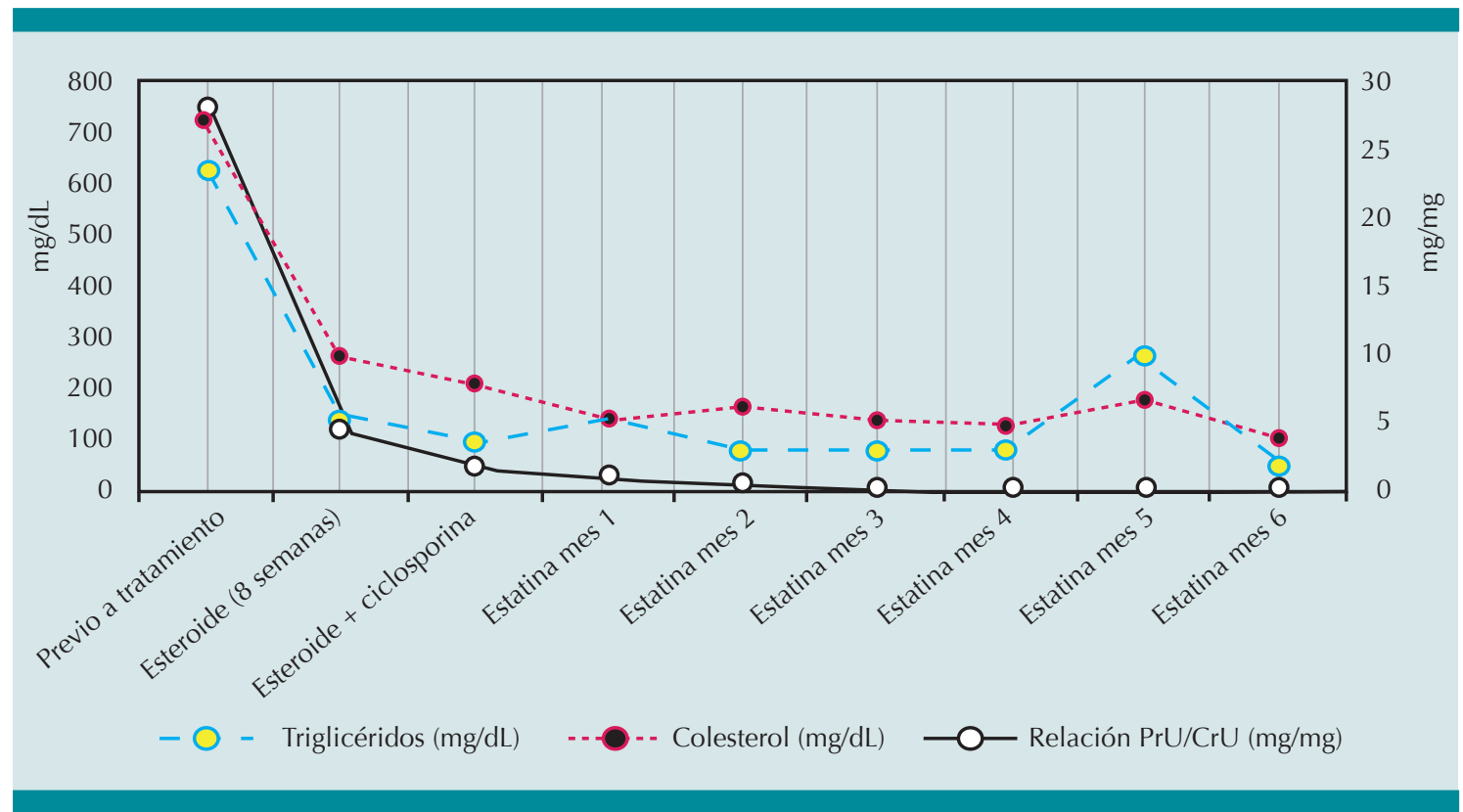

Figura 1. Evolución del caso clínico 1.

y excreción de proteínas en orina de $342 \mathrm{mg} /$ kg/día (PrU/CrU $40.88 \mathrm{mg} / \mathrm{mg}$ ). Se reinició prednisona a dosis de $60 \mathrm{mg} / \mathrm{m}^{2} \mathrm{SC} /$ día durante ocho semanas. Debido a la falta de respuesta se agregó al tratamiento ciclosporina $(5 \mathrm{mg} / \mathrm{kg} /$ día). Durante el primer mes de tratamiento no presentó variación en los valores de laboratorio de lípidos, albúmina sérica o proteinuria. Previo consentimiento informado y firma del mismo por los padres, se inició simvastatina $5 \mathrm{mg}$ al día con lo que hubo, al primer mes de tratamiento, disminución de colesterol (535 mg/dL), triglicéridos (794 mg/dL) y de la proteinuria (82 mg/kg/día). A las 12 semanas de tratamiento con simvastatina 
se alcanzó remisión parcial del síndrome nefrótico con albúmina sérica de $2.5 \mathrm{~g} / \mathrm{dL}$, colesterol $464 \mathrm{mg} / \mathrm{dL}$, triglicéridos $595 \mathrm{mg} / \mathrm{dL}$, proteinuria de $3.2 \mathrm{mg} / \mathrm{kg} / \mathrm{día} \mathrm{(PrU/CrU} 0.7 \mathrm{mg} / \mathrm{mg}$ ). Durante el seguimiento presentó recaída en el síndrome nefrótico en dos ocasiones, al cuarto y décimo meses de tratamiento; ambas asociadas con infección de vías respiratorias sin requerir suspender inmunosupresor ni hipolipemiante (Cuadro 2).

Completó 64 semanas de tratamiento con ciclosporina, simvastatina y prednisona en esquema de reducción; con lo que alcanzó remisión del síndrome nefrótico. Se ha mantenido sin tratamiento desde entonces sin recaídas; actualmente con albúmina sérica de $4.2 \mathrm{~g} / \mathrm{dL}$, colesterol $185 \mathrm{mg} / \mathrm{dL}$, triglicéridos $140 \mathrm{mg} / \mathrm{dL}$, proteinuria negativa, sin elevación significativa de la creatina fosfoquinasa respecto a valores basales, sin efectos adversos asociados al tratamiento y función renal conservada (Figura 2).

\section{DISCUSIÓN}

El síndrome nefrótico causa la pérdida urinaria de proteínas. La hiperlipidemia se encuentra invariablemente en la mayoría de los pacientes con síndrome nefrótico. El uso de inhibidores de la HMC-CoA reductasa (estatinas) ha demostrado ser eficaz en la reducción de los niveles séricos de colesterol. ${ }^{13}$

En los casos descritos el uso de simvastatina en uno de los pacientes y de atorvastatina en el otro se debió principalmente a la disponibilidad del medicamento. Las estatinas, comparadas con otros agentes hipolipemiantes, han demostrado una reducción consistente en los niveles de colesterol, además tienen efecto en los niveles de triglicéridos. ${ }^{14}$

Sanjad y sus colaboradores reportaron, en pacientes pediátricos con síndrome nefrótico corticorresistente, una reducción de $32 \%$ en el colesterol total al segundo mes de tratamiento con estatinas. El efecto máximo en los valores de triglicéridos se alcanzó al cuarto mes de tratamiento con una reducción de $41.43 \% .^{15}$

En un estudio en el que se evaluó el efecto de la dieta y simvastatina, en pacientes con síndrome nefrótico corticorresistente, se reportó una reduc-

Cuadro 2. Valores de laboratorio del caso clínico 2

\begin{tabular}{|c|c|c|c|c|c|}
\hline & $\begin{array}{l}\text { Relación PrU/ } \\
\text { CrU (mg/mg) }\end{array}$ & $\begin{array}{l}\text { Proteinuria } \\
\text { (mg/kg/día) }\end{array}$ & $\begin{array}{c}\text { Albúmina sérica } \\
(\mathrm{g} / \mathrm{dL})\end{array}$ & $\begin{array}{l}\text { Triglicéridos } \\
\text { (mg/dL) }\end{array}$ & $\begin{array}{c}\text { Colesterol } \\
(\mathrm{mg} / \mathrm{dL})\end{array}$ \\
\hline Previo a tratamiento & 40.88 & 342 & 0.8 & 2834 & 2566 \\
\hline Esteroide (8 semanas) & 47.22 & 385 & 1.2 & 1755 & 832 \\
\hline Esteroide + ciclosporina & 44.57 & 365 & 1 & 1929 & 696 \\
\hline Estatina mes 1 & 15.3 & 82.2 & 1.2 & 794 & 535 \\
\hline Estatina mes 2 & 16.7 & 20 & 1.5 & 746 & 580 \\
\hline Estatina mes 3 & 0.7 & 3.2 & 2.5 & 595 & 464 \\
\hline Estatina mes 4 (recaída) & 15.8 & 134.5 & 1.2 & 872 & 666 \\
\hline Estatina mes 8 & 0.59 & 6.4 & 2.2 & 294 & 444 \\
\hline Estatina mes 10 (recaída) & 22.66 & 197.3 & 1 & 428 & 486 \\
\hline Estatina mes 16 & 0.05 & 0.4 & 2.9 & 408 & 411 \\
\hline $\begin{array}{l}\text { Suspensión de tratamiento } \\
\text { (mes 18) }\end{array}$ & 0 & 0 & 3.8 & 211 & 273 \\
\hline Mes 28 & 0 & 0 & 4.2 & 140 & 185 \\
\hline
\end{tabular}




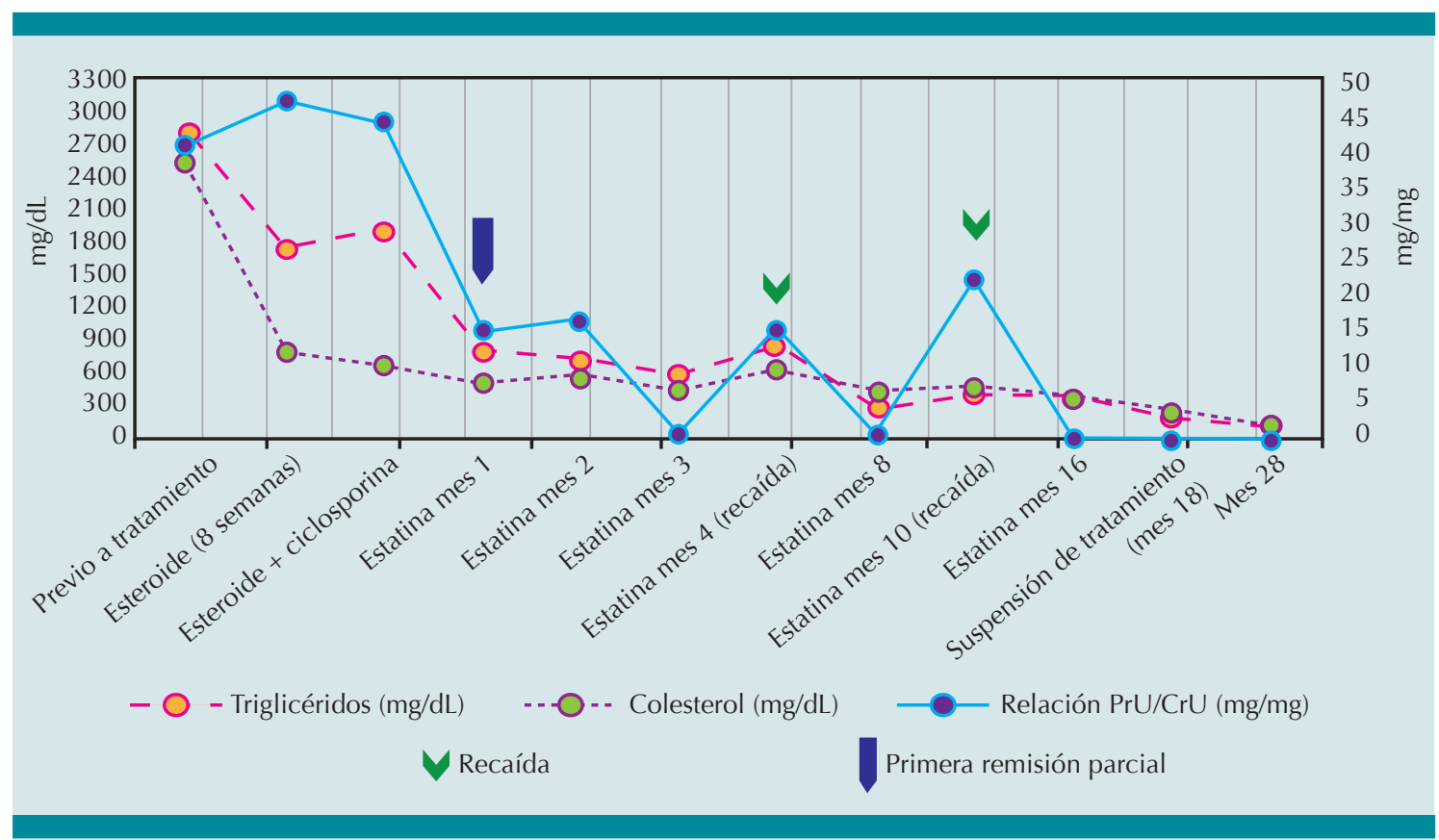

Figura 2. Evolución del caso clínico 2.

ción de 30\% en el segundo mes con simvastatina comparada con los valores basales (solo dieta) de colesterol; no se encontró disminución significativa en los niveles de trigliceridos. ${ }^{16}$ La albúmina sérica en los estudios previamente mencionados se mantuvo sin variación. Los valores de proteinuria tampoco presentaron cambio en dichos reportes. ${ }^{15,16}$

En siete pacientes con síndrome nefrótico primario y tratamiento con lovastatina se logró una disminución significativa en los niveles de colesterol, apolipoproteina B y triglicéridos a 2 años de seguimiento, así como disminución significativa de la proteinuria en 4 de estos pacientes con tratamiento concomitante con inhibidores de la enzima convertidora de angiotensina. ${ }^{17}$

En nuestros dos pacientes se observó efecto hipolipemiante desde el primer mes de inicio con estatinas; de forma similar a lo encontrado en la literatura los niveles de lípidos no siempre llegan a niveles normales a pesar de una disminución significativa en la proteinuria.

La remisión del síndrome nefrótico corticorresistente es alcanzada hasta en un $70 \%$ de los pacientes a las 26 semanas de tratamiento con ciclosporina, aunque se ha reportado respuesta desde el primer mes de tratamiento. ${ }^{18,19}$

Se ha reportado hiperlipidemia inducida por el uso prolongado de ciclosporina en seres humanos; en ratas Sprage-Dawley se demostró que este efecto es debido a una alteración en la expresión de la enzima colesterol-7 $\alpha$ hidroxilasa. $^{20}$

La hiperlipidemia asociada con el síndrome nefrótico afecta la farmacocinética y disminuye el efecto inmunosupresor de la ciclosporina. ${ }^{21} \mathrm{Se}$ ha reportado la probable mejoría de la farmacocinética de la ciclosporina con el tratamiento concomitante con pravastatina, lo que llevó a la 
remisión del síndrome nefrótico en una paciente en Japón; ${ }^{22}$ de forma similar a lo descrito en el caso clínico número dos no se había alcanzado remisión del síndrome nefrótico hasta el inicio del tratamiento con estatinas. Sin embargo, el efecto de los hipolipemiantes sobre el grado de proteinuria no se ha demostrado en estudios controlados aleatorizados. ${ }^{23}$

El uso de estatinas en pacientes con síndrome nefrótico resistente a esteroides es una terapéutica prometedora; es necesario realizar mayor número de estudios, aumentar el número de pacientes y el tiempo de seguimiento para evaluar si existe disminución en el número de recaídas; en la sobrevida y progresión a enfermedad renal crónica, así como el riesgo cardiovascular y realizar una recomendación acertada respecto a su uso en pacientes pediátricos con síndrome nefrótico.

\section{REFERENCIAS}

1. Avner ED, Harmon WE, Niaudet P, Yoshikawa N. Pediatric nephrology. 6th ed. Berlin: Springer; c 2009. 667p.

2. Peña A, Bravo J, Melgosa M, Fernández C, Meseguer C, Espinoza L, et al. Steroid-resistant nephrotic syndrome: long-term evolution after sequential therapy. Pediatr Nephrol. 2007;22(11):1875-1880.

3. Loeffler K, Gowrishankar M, Yiu V. Tacrolimus therapy in pediatric patients with treatment-resistant nephrotic syndrome. Pediatr Nephrol. 2004;19(3):281-287.

4. Niaudet P. Treatment of childhood steroid-resistant idiopathic nephrosis with a combination of cyclosporine and prednisone. French Society of Pediatric Nephrology. J Pediatr. 1994;125(6 Pt 1):981-986.

5. Hodson EM, Willis NS, Craig JC. Interventions for idiopathic steroid-resistant nephrotic syndrome in children. Cochrane Database Syst Rev. 2010 Nov; 10(11):CD003594. doi: 10.1002/14651858.CD003594.pub4.

6. Kaysen GA, Davies RW. Reduction in proteinuria attenuates hyperlipidemia $\mathrm{n}$ the nephrotic syndrome. J Am Soc Nephrol. 1990;1(5 Suppl 2):S75-S79.

7. Kaysen GA, Gambertoglio J, Felts J, Hutchison FN. Albumin synthesis, albuminuria and hyperlipemia in nephrotic patients. Kidney Int. 1987;31(6):1368-1376.

8. Thabet MA, Salcedo JR, Chan JC. Hyperlipidemia in childhood nephrotic syndrome. Pediatr Nephrol. 1993;7(5):559566.
9. Querfeld U. Should hyperlipidemia in children with the nephrotic syndrome be treated? Pediatr Nephrol. 1999;13(1):77-84.

10. Duplaga BA. Treatment of childhood hypercholesterolemia with HMG-CoA reductase inhibitors. Ann Pharmacother. 1999;33(11):1224-1227.

11. García-de-la-Puente S. Arredondo-García JL, GutiérrezCastrellón P, Bojorquez-Ochoa A, Maya ER, Pérez-Martínez Mdel P. Efficacy of simvastatin in children with hyperlipidemia secondary to kidney disorders. Pediatr Nephrol. 2009;24(6):1205-1210. doi: 10.1007/s00467-099-1128-7.

12. Buemi M, Nostro L, Crasci E, Barillà A, Consentino V, Aloisi $C$, et al. Statins in nephrotic syndrome: a new weapon against tissue injury. Med Res Rev. 2005;25(6):587-609.

13. Endo A. The Discovery and development of HMC-CoA reductase inhibitors. J Lipid Res. 1992;33(11):1569-1582.

14. Massy ZA, Ma JZ, Louis TA, Kasiske BL. Lipid-lowering therapy in patients with renal disease. Kidney Int. 1995;48(1):188-198.

15. Sanjad SA, al-Abbad A, al-Shorafa S. Management of hyperlipidemia in children with refractory nephrotic syndrome: the effect of statin therapy. J Pediatr. 1997;130(3):470-474.

16. Coleman JE, Watson AR. Hyperlipidaemia, diet and simvastatin therapy in steroid-resistant nephrotic syndrome of childhood. Pediatr Nephrol. 1996;10(2):171-174.

17. Prata MM, Noqueira AC, Pinto JR, Correira AM, Vicente $\mathrm{O}$, Rodrigues $\mathrm{MC}$, et al. Long-term effect of lovastatin on lipoprotein profile in patients with primary nephrotic syndrome. Clin Nephrol. 1994;41(5):277-283.

18. Ponticelli C, Rizzoni G, Edefonti A, Altieri P, Rivolta E, Rinaldi S, el al. A randomized trial of cyclosporine in steroid-resistant idiopathic nephrotic syndrome. Kidney Int. 1993;43(6):1377-1384.

19. Cattran DC, Appel GB, Hebert LA, Hunsicker LG, Pohl MA, Hoy WE, et al. A randomized trial of cyclosporine in patients with steroid-resistant focal segmental glomerulosclerosis. North America Nephrotic Syndrome Study Group. Kidney Int. 1999;56(6):2220-2226.

20. Vaziri ND, Liang K, Azad H. Effect of cyclosporine on HMGCoA reductase, cholesterol 7alpha-hydroxylase, $L D L$ receptor, HDL receptor, VLDL receptor, and lipoprotein lipase expressions. J Pharmacol Exp Ther. 2000;294(2):778-783.

21. Hirano T, Kawamura T, Fukuda S, Kohsaka S, Yoshikawa N, Yoshida M, et al. Implication of cholesterol in cyclosporine pharmacodynamics in minimal change nephrotic syndrome. Clin Pharmacol Ther. 2003;74(6):581-590.

22. Ito S, Machida H, Inaba A, Harada T, Okuyama K, Nakamura $\mathrm{T}$, et al. Amelioration of steroids and cyclosporine-resistant nephrotic syndrome by pravastatin. Pediatr Nephrol. 2007;22(4):603-606.

23. Kong $X$, Yuan H, Fan J, Li Z, Wu T, Jiang L. Lipid-lowering agents for nephrotic syndrome. Cochrane Database Syst Rev. 2013;10(12):CD005425. doi: 10.1002/14651858. cd005425.pub2. 